\title{
Políticas de juventud, adolescencia y salud
}

\author{
Dina Krauskopf*
}

\begin{abstract}
Resumen
Se revisa la evolución de los paradigmas de la salud y de las políticas de juventud a la luz de la modernización, globalización y exclusión. Se analizan los modelos que derivan de los enfoques tradicionales, reduccionistas y avanzados, el problema de la condición sociojurídica en la adolescencia y juventud, la reformulación del concepto de riesgo y protección, la diferencia entre promoción de la salud y prevención de la enfermedad. Se consideran enfoques avanzados para la construcción de políticas en salud.
\end{abstract}

Palabras clave:Juventud, riesgo, salud, ciudadanía, paradigmas, políticas.

\begin{abstract}
Evolution of health and youth policy paradigms is revised under the lens of modernization, globalization and exclusion. Analysis are done over models derived form traditional, reductionism and innovative considerations; studying issues of socio-juridical conditions in adolescence and youth; reformulating concepts of risk and protectio n;distinguishing between promoting health and preventing disease. Advanced approaches are considered for the creation of health policies.
\end{abstract}

Key words: Youth, risk, health, citizenship,paradigms, policies.

* Profesora Emérita Universidad de Costa Rica. Consultora Internacional en Políticas de Adolescencia y Juventud. Master en Psicología Clínica. dinakr@racsa.co.cr 
Los cambios epocales constituyen un trasfondo clave para el surgimiento de políticas públicas de adolescencia y juventud. El modelo económico globalizado ha modificado el mercado laboral y contribuye al incremento de la brecha social al interior de los países latinoamericanos; ya no puede desconocerse que sus juventudes son heterogéneas y están expuestas a diversos grados de exclusión. Por otro lado, la modernización ha contribuido a la prolongación de la vida. Todo ello impacta tanto el posicionamiento juvenil como la situación adulta en las sociedades del siglo XXI.

La fase juvenil ocupa, en el ciclo vital, un número creciente de años; la pubertad se da a edades más tempranas y la vida adulta se prolonga en el contexto de la velocidad de los cambios, por lo que ya no es un período estable, sino que está sujeta a importantes transformaciones. La flexibilidad juvenil para incorporar los conocimientos y aportar a la innovación se valoriza. Las juventudes contemporáneas se afirman cada vez más en sus identidades juveniles.

Sin la inclusión de las juventudes contemporáneas, no es posible la democracia ni el desarrollo equitativo de las sociedades. Con el fin de avanzar en su incorporación, se han elaborado, en diversos países de América Latina, Políticas de Juventud, Planes de Acción y Programas de Salud (si bien estos últimos están más orientados a la adolescencia que a la juventud). Las Leyes de Juventud creadas son un instrumento que las respalda y los sistemas de juventud han favorecido la construcción conjunta de políticas entre los entes gubernamentales y las juventudes. La Convención de los Derechos del Niño y, recientemente, la Convención Iberoamericana de los Derechos de los Jóvenes dan un marco de referencia fundamental a estas tendencias.

El avance de la atención de la salud para la adolescencia ha sido vigoroso en los últimos tiempos. Enfrenta aún desafíos considerables para alcanzar el lugar requerido en los servicios y políticas. Su desarrollo ha permitido confrontar enfoques conceptuales 
y metodológicos. Ha demandado reconocer que los paradigmas tradicionales eran insuficientes para integrar al desarrollo de sus sociedades y fomentar la calidad de vida de las personas en edades estratégicas del ciclo vital. El abordaje adecuado de esta población ha mostrado también la estrechez de un enfoque puramente sectorial en la atención de su salud y ha promovido alianzas estratégicas que amplían los escenarios de las intervenciones, así como perspectivas que demandan la articulación de políticas que sustenten estos programas.

El presente trabajo revisa esta problemática y procura sistematizar los referentes clave en la construcción de las políticas y programas dirigidos a la salud de la adolescencia y juventud.

\section{La edad en las políticas y programas de Adolescencia y Juventud}

El referente etáreo es un elemento imprescindible (aunque en ningún caso suficiente) en la definición de los grupos meta de políticas y programas. Sin embargo, en el caso de las políticas públicas de juventud, es un factor que conspira contra la necesaria articulación y cobertura.

En el campo normativo, la Convención de los Derechos del Niño constituye un avance al aplicar el enfoque de los derechos a las personas hasta los 18 años, si bien cabe destacar que no se menciona a los adolescentes. Tal situación se modifica cuando se crean, en el marco de la Doctrina de la Protección Integral, instrumentos legales derivados de la mencionada Convención, como la Ley de Justicia Penal Juvenil y los códigos de los Derechos de la Niñez y la Adolescencia.

El sujeto juvenil comparte la condición socio-jurídica con la población menor de 18 años, pero, a partir de este límite, generalmente ha adquirido el status de la ciudadanía formal. Desde los 18 años (por alcanzar legalmente la mayoría de edad), las personas son juzgadas como adultas, reflejando la ambigüedad del 
sujeto juvenil y favoreciéndose su invisibilización en la programación social. Es bastante común que quede subsumido o desvanecido en las propuestas, bajo la categoría de adulto. Así, las particularidades de este grupo tienden a diluirse en un diseño de política social, económica y laboral dirigida al conjunto de la población adulta sin distingo alguno (Krauskopf y Mora, 2000). Por lo tanto, es importante reconocer que, pese a los avances en el plano normativo de los derechos y en el desarrollo de algunos programas, se aprecian inconsistencias asociadas al carácter dual del sujeto juvenil, que fragmentan el reconocimiento de sus identidades y debilitan la atención a su desarrollo social.

El planteamiento de políticas de juventud contempla el segmento poblacional que promedia sus límites cronológicos entre los 18 y los 30 años (en América Latina, se encuentran límites entre 12 y 35 años), aunque la oferta pública en salud está principalmente dirigida al período adolescente. La existencia de programas de adolescencia contribuye al desarrollo de la juventud, pero no alcanza períodos cruciales de la vida de las personas jóvenes, las que, en el campo de la salud, pasan a ser incorporadas al segmento adulto. Las categorías de la OMS contemplan a la juventud, si bien presentan una superposición etárea, pues se plantea que la adolescencia va de los 10 a los 19 y la juventud de los 15 a los 24 . El quinquenio entre 15 y 19 cae bajo ambas denominaciones. En estas condiciones, la juventud engloba la adolescencia, pero la adolescencia no engloba a la juventud, lo que tiene consecuencias sociopolíticas. La Convención Iberoamericana de los Derechos de los Jóvenes ubica al sujeto juvenil entre los $15 \mathrm{y}$ los 24 años.

Últimamente en Costa Rica se reconoce la necesidad de ampliar el grupo meta de su Programa de Atención Integral a la Salud en la Adolescencia a los 24 años, 11 meses, aun cuando ello no se ha implementado. Las bases del Programa señalan la importancia del grupo de 20 a 24 años: “...en su gran mayoría, parecen no haber podido finiquitar las tareas propias de la adolescencia 
que, se supone, marcan el término de esta etapa del ciclo de vida y comparte muchas de las necesidades de los menores de 19 años, no encontrando respuestas a estas, en los Programas de Salud del Adulto" (CCSS, 2002).

\section{El reconocimiento del sujeto juvenil en las políticas}

La ausencia de una programación social que integre articuladamente la fase juvenil puede ser considerada parte de una crisis social que incluye la fractura de los paradigmas y supuestos que sostiene el modelo de juventud. Las políticas que conciernen a la juventud se han expresado tanto en omisiones como en afirmaciones y en políticas implícitas.

Hemos distinguido tres tipos de políticas de acuerdo a los paradigmas que las sostienen: 1) tradicionales, que enfocan el período juvenil como una transición hacia la adultez, meta de su preparación; 2) reduccionistas, donde aparece el sujeto juvenil como portador de problemas que amenazan el orden social o como objeto de asistencia por el déficit en su desarrollo; 3) avanzadas, que se orientan al empoderamiento de los jóvenes como ciudadanos y actores estratégicos del desarrollo (Krauskopf, 2003).

A continuación, analizaremos cada paradigma y presentaremos la sistematización de su relación con las políticas y los programas de adolescencia y juventud y la atención en salud.

\subsection{La adolescencia y juventud como período preparatorio en las políticas}

La juventud entendida como un período de transición entre la niñez y la adultez lleva a dar centralidad al enfoque de preparación en las políticas tradicionales de adolescencia y juventud. Es el único período del ciclo vital definido como transición, lo que le niega sus particularidades como sujeto social. Desde dicha 
perspectiva, la política por excelencia ha sido la Educación. El futuro sería el eje orientador justificando la idea de la moratoria psicosocial $^{1}$ como el mayor beneficio concedido por la sociedad al período juvenil, con lo cual no se visualiza a las juventudes como sujeto social activo en su presente. La representación social del tiempo libre juvenil se dirige a un imaginario constituido por aquellos sectores de la juventud que están en capacidad de vivir la moratoria.

Cuadro 1. Políticas tradicionales: Paradigma de preparación

\begin{tabular}{|l|l|l|l|}
\hline Paradigma & $\begin{array}{l}\text { Estrategias de } \\
\text { Políticas }\end{array}$ & Programas & Atención \\
\hline $\begin{array}{l}\text { Transición a la } \\
\text { transición a la } \\
\text { adultez }\end{array}$ & $\begin{array}{l}\text { Sectoriales } \\
\text { Universales } \\
\text { Extensión de } \\
\text { la cobertura } \\
\text { educativa }\end{array}$ & $\begin{array}{l}\text { Homogéneos } \\
\text { Aislados }\end{array}$ & $\begin{array}{l}\text { Indiferenciada } \\
\text { Protección } \\
\text { Externa }\end{array}$ \\
$\begin{array}{l}\text { Etapa de } \\
\text { crecimiento y } \\
\text { preparación para } \\
\text { la adultez }\end{array}$ & $\begin{array}{l}\text { Tiempo libre } \\
\text { recreativo con } \\
\text { baja cobertura } \\
\text { Servicio militar }\end{array}$ & $\begin{array}{l}\text { Sujetos pasivos } \\
\text { de servicios y } \\
\text { programas } \\
\text { Discriminación } \\
\text { etárea }\end{array}$ \\
\hline
\end{tabular}

Fuente: Dina Krauskopf. Nueva Sociedad \#200.2005. Versión modificada

Los fundamentos del paradigma tradicional de preparación no reconocen el hecho actual de un futuro incierto, ni tampoco que, en los estratos pobres de la región, frecuentemente se encuentra la premura psicosocial, la que no espera más allá de los cambios hormonales de la pubertad para las responsabilidades de automantención y mantención familiar. Tampoco se incorpora el hecho de que, con la globalización y la modernización, el conocimiento y la flexibilidad para los aprendizajes pasaron a ser

1 Erikson (1974) establece la centralidad de la moratoria psicosocial para la elaboración de la identidad en el período juvenil. La considera una parte constitutiva de su preparación a través de la postergación de la acción y la toma de decisiones. Es un tiempo de ensayo y error que permite probar diversos roles y orientaciones en una estructura de oportunidades que facilita la paulatina integración de las identificaciones infantiles y la elaboración final de la identidad. 
sustantivos y son, precisamente, el punto fuerte de las capacidades juveniles. Los cambios posicionan de modo distinto el rol y la jerarquía de la experiencia, lo que conduce a que el modelo adulto contemporáneo deba dejar de ser considerado un parámetro suficiente para orientar la preparación.

Entre las políticas omisas que se derivan de dicho paradigma, está la programación en Salud orientada a la morbimortalidad de adultos y niños, con una atención indiferenciada para adolescentes y jóvenes que los discrimina y las políticas de Trabajo orientadas a adultos. Los jóvenes que no estudian ni trabajan constituyen la generación $\mathrm{NET}^{2}$ que reclama una programación social integradora, un replanteamiento del modelo educativo y un desafío para la programación que atiende la salud adolescente y juvenil.

Una política implícita para la juventud es el servicio militar, preparatorio de destrezas específicas para el cumplimiento de deberes de responsabilidad y unidad nacional, con formas autoritarias de integración y efectos discutibles en el desarrollo juvenil. El ingreso obligatorio interrumpe los estudios y las secuelas de los conflictos bélicos han implicado diversos problemas específicos para el desarrollo juvenil y su salud.

\subsection{La adolescencia y juventud como etapa problema en las políticas}

Ante la ausencia de la visibilización del sujeto juvenil en su tránsito hacia la adultez, los jóvenes solo emergen como tales cuando perturban el orden social. La juventud como etapa problema se enfoca desde políticas reduccionistas, sectoriales y compensatorias. Los grupos meta relevantes se encuentran en la juventud urbana popular y la atención se presta a problemas específicos.

2 Denominación aportada por el investigador argentino Sergio Balardini en el Seminario Euro latinoamericano de Estudios de Juventud: Los Jóvenes y la Sociedad de Información. Globalización y Antiglobalización en Europa y América Latina. Lleida-Barcelona, mayo, 2002. 
Cuadro 2. Políticas reduccionistas: Paradigma de Etapa Problema

\begin{tabular}{|c|c|c|c|}
\hline Paradigma & \begin{tabular}{|l} 
Estrategias de \\
Políticas \\
\end{tabular} & Programas & Atención \\
\hline $\begin{array}{l}\text { Riesgo y } \\
\text { trasgresión } \\
\text { Etapa problema } \\
\text { para la sociedad }\end{array}$ & $\begin{array}{l}\text { Compensatorias } \\
\text { Sectoriales } \\
\text { (Predominante- } \\
\text { mente salud y } \\
\text { justicia) } \\
\text { Focalizadas } \\
\text { Fragmentadas }\end{array}$ & $\begin{array}{l}\text { Marco legal } \\
\text { tutelar a menores } \\
\text { en abandono, } \\
\text { deambulación } \\
\text { delincuencia } \\
\text { Criminalización } \\
\text { de la juventud en } \\
\text { pobreza } \\
\text { Relevancia a } \\
\text { juventud urbano } \\
\text { popular } \\
\text { Dispersión de las } \\
\text { ofertas }\end{array}$ & $\begin{array}{l}\begin{array}{l}\text { Problemas } \\
\text { sintomáticos }\end{array} \\
\text { Asistencialidad } \\
\text { Prevención } \\
\text { Rehabilitación } \\
\text { Control externo }\end{array}$ \\
\hline
\end{tabular}

Fuente: Dina Krauskopf. Nueva Sociedad \#200.2005. Versión modificada

Fueron los problemas de salud sexual y reproductiva los primeros en poner a la adolescencia en el tapete como sujeto prioritario de atención de la salud. Así, las mujeres adolescentes entraron en las políticas del sector salud a partir de sus embarazos. Se fueron adicionando temas que enmarcan restringida y negativamente la vida juvenil: la delincuencia, las drogas, la deserción escolar, las maras o bandas, etc.

Se descubre que una impactante proporción de las muertes durante la adolescencia se producen por las llamadas causas externas $y$, por lo tanto, prevenibles. Se modifica así el paradigma que establecía la equivalencia "adolescencia = edad más sana" por el paradigma "adolescencia = etapa de riesgo" y se focaliza la atención de los adolescentes y jóvenes de acuerdo al problema específico de que son portadores.

Desde este paradigma, la causa última de las "patologías" juveniles se identificó en el sujeto juvenil, su propia conducta es considerada un factor de riesgo, de ahí que la intervención priorizó la acción en él y descuidó el contexto. A partir de los polos sinto- 
máticos y problemáticos, se construyó en la sociedad una percepción generalizadora de la juventud que la estigmatiza y su visibilización es negativa. Es lo que en CIDPA llaman los cuatro jinetes del Apocalipsis: drogas, alcohol, violencia y promiscuidad (Oyarzún, 2006). La estigmatización criminalizante genera un círculo vicioso al fomentar en los jóvenes un empoderamiento aterrante para la sociedad que los excluye (Krauskopf, 1996).

Los programas son orientados a los comportamientos de riesgo y trasgresión, su asistencia y prevención, así como también existen programaciones que se inspiran en matrices de corte autoritario para la rehabilitación de jóvenes y abogan por incrementar los mecanismos de control y represión social para protección de la sociedad. Aquí se encuentran las medidas que Santa Cruz (2003) denomina como Populismo Punitivo. En países donde domina esta visión, parece darse un debilitamiento de las posibilidades de desarrollar estrategias sostenibles para la implementación de políticas avanzadas de juventud.

El abordaje sectorial y fragmentado que los programas han desarrollado desde este paradigma ha demostrado su ineficacia (Blum, 1996) y existen iniciativas que procuran construir perspectivas más integrales. Es necesario incorporar los avances en el campo del derecho, valorar los análisis socioeconómicos de los comportamientos de riesgo, la grupalidad juvenil y la profundización sistémica sobre la violencia. Antes de pasar a los paradigmas de las políticas avanzadas, señalaremos algunos avances en la conceptualización del riesgo.

\subsubsection{La reformulación social del concepto de riesgo}

El concepto de riesgo, tan estrechamente asociado a la vida juvenil, ha experimentado una reformulación psicosocial. Jessor (1991) destaca que no pueden valorarse las conductas de riesgo si no se hace un análisis de costo beneficio de la conducta tomando en cuenta las condiciones que se dan a la juventud. La posibilidad de que los adolescentes abandonen un comportamiento 
riesgoso está relacionada fuertemente con la posibilidad de que se les ofrezcan alternativas de satisfacción valiosas para ellos.

Beck (1998) señala que, como consecuencia de los procesos de modernización, las sociedades contemporáneas se han constituido en sociedades de riesgo. La desigualdad se expresa en el reparto de riqueza y el reparto de riesgos. Los peligros producidos socialmente sobrepasan la seguridad y el impacto diferencial de la brecha social lleva a que la riqueza se acumule en los estratos más altos, mientras que los riesgos se acumulan en los más bajos.

Desde estas perspectivas, queda claro que, para la comprensión del comportamiento de riesgo, el empoderamiento aterrante y problemas sociales como la violencia, es importante considerar lo que hemos llamado el riesgo estructural, cuyas características se desglosan en el cuadro siguiente.

\section{Cuadro 3. Riesgo estructural}

- Entornos marginalizados, desprotegidos, bélicos y criminalizados

- Territorios donde la identidad positiva del colectivo es denegada socialmente

- La estigmatización de la exclusión y violencia juvenil como delincuencia

- La calle como espacio fundamental de socialización y aprendizaje

- La re-socialización en la cárcel

- Carencia de espacios para la incorporación de habilidades y destrezas

- Carencia de espacios para el progreso de la convivencia, la calidad de vida y la incorporación social

- Carencia de espacios para expresiones de creatividad y participación juvenil legitimada

- Escasa presencia de instituciones

- Falta de acceso a servicios públicos

- Desconfianza interpersonal e institucional

- Zonas con mayor exposición a riesgo de la vida

- Condiciones de hacinamiento

- Trabajos marginales

- Menos oportunidades de gratificación y mayor oferta de espacios como bares, billares, acceso a alcohol y drogas

Fuente: Krauskopf, Dina (2003). Dimensiones de la Violencia. Versión modificada 
Se trata, por lo tanto, de espacios físicos donde, al decir de Beck, se acumulan los riesgos tanto a nivel de las representaciones sociales como en las condiciones concretas de convivencia y acceso a oportunidades de protección, atención y desarrollo. Diversos estudios muestran que, en este contexto, no puede prevalecer la visión de riesgo como un comportamiento individual y se requieren políticas para reducir la pobreza, aumentar el capital social en estos territorios y tomar en cuenta constructivamente a la juventud (ERIC, IDESO, IDIES, IUODP, 2004).

\subsection{La juventud ciudadana como sujeto de políticas}

Desde el paradigma de la ciudadanía, las personas jóvenes dejan de ser definidas por sus incompletitudes y sintomas, para ser consideradas sujetos de derechos. Ya no se trata solo de la ciudadanía formal, de ejercer el derecho al voto, sino que se da centralidad a la ciudadanía integral de niños, adolescentes y jóvenes. La construcción de políticas con este marco destaca la participación juvenil y el reconocimiento de la fase juvenil como un importante período de desarrollo social.

Cuadro 4. Políticas avanzadas: paradigma de ciudadanía

\begin{tabular}{|c|c|c|c|}
\hline Paradigma & $\begin{array}{l}\text { Estrategias de } \\
\text { Políticas }\end{array}$ & Programas & Atención \\
\hline $\begin{array}{l}\text { Juventud } \\
\text { ciudadana } \\
\text { Etapa de } \\
\text { crecimiento } \\
\text { y desarrollo } \\
\text { social }\end{array}$ & $\begin{array}{l}\text { Rectoría de } \\
\text { juventud en } \\
\text { política pública } \\
\text { Intersectorialidad } \\
\text { Priorizan la } \\
\text { inclusión y } \\
\text { participación de } \\
\text { jóvenes como } \\
\text { sujetos explícitos } \\
\text { de derechos } \\
\text { políticos, civiles, } \\
\text { culturales, sociales } \\
\text { y económicos }\end{array}$ & $\begin{array}{l}\text { Integrales, } \\
\text { participativos } \\
\text { extensión de } \\
\text { alianzas } \\
\text { Equidad de } \\
\text { género, cultura, } \\
\text { residencia } \\
\text { urbano-rural, } \\
\text { estrato socio- } \\
\text { económico } \\
\text { Empodera- } \\
\text { miento }\end{array}$ & $\begin{array}{l}\text { Protección Integral } \\
\text { Reparación } \\
\text { Promoción } \\
\text { Prevención } \\
\text { Atención integral } \\
\text { Acceso igualitario } \\
\text { Exigibilidad de } \\
\text { derechos } \\
\text { Escenarios diversos } \\
\text { Manejo social del } \\
\text { riesgo } \\
\text { Colaboración } \\
\text { intergeneracional }\end{array}$ \\
\hline
\end{tabular}

Fuente: Dina Krauskopf. Nueva Sociedad \#200.2005. Versión modificada 
Los programas de salud que se han desarrollado en las últimas décadas en América Latina han ido incrementando la inclusión de adolescentes y jóvenes con la participación, llamada protagónica, como una forma de resaltar su visibilización positiva. Igualmente, se ha enriquecido la visión de la protección. Tradicionalmente, esta es vista como producto de la acción del otro, la prevención como un control externo dirigido a reducir y limitar los factores de daño. Desde la Doctrina de la Protección Integral, se protege brindando protección de los derechos, en lugar de proteger a los niños y adolescentes en tanto personas como lo plantea la Doctrina del Menor Irregular. Se trata de una protección y prevención apoyada en el empoderamiento que deriva de la promoción de condiciones para la capacidad de decisión y de participación. La protección social debe reconocer que el retiro de respuestas inadecuadas requiere ir acompañado de la oferta de un replanteamiento de la percepción de los problemas y opciones, junto con respuestas más satisfactorias a las necesidades de las poblaciones vulnerables. Además, se considera la restitución de los derechos que han sido violados, lo que desemboca, por lo tanto, en acciones reparatorias.

El enfoque integral de la salud adolescente y juvenil da prioridad a la responsabilidad de exigir y construir el derecho a la salud plena; la articulación de políticas; la legislación y los programas intersectoriales en el fomento de la salud; la promoción de la salud en los diferentes escenarios; el fortalecimiento de la prevención primaria de las enfermedades y daños; el enfrentamiento equitativo de las condiciones de género, cultura, residencia ruralurbana, estrato socioeconómico; el reconocimiento de las capacidades de adolescentes y jóvenes, sus familias y comunidades de aportar a la salud, la cultura y el desarrollo. Los lineamientos dan un lugar decisorio a la participación social; la visibilización y legitimación de los logros de adolescentes y jóvenes; el fomento de nuevas relaciones intergeneracionales así como entre prestadores de servicios, adolescentes y jóvenes: sus derechos son el 
centro. En consecuencia, es estratégica la visibilización de obstáculos al desarrollo de la salud de adolescentes y jóvenes para la movilización en defensa de los derechos vulnerados.

La acumulación de experiencias y la sistematización de conocimientos evidencian que la implementación de este paradigma requiere precisiones en el concepto de participación para ir de la apariencia a la efectividad y al desarrollo de estrategias intergeneracionales de colaboración (Krauskopf, 2003). En las formas contemporáneas de participación juvenil, prevalecen los parámetros éticos, estéticos, subjetivos, la valoración de las metas palpables por sobre los parámetros político-ideológicos de épocas pasadas (Serna, 1998). La extensión de las alianzas ha permitido la búsqueda de articulaciones intersectoriales y la estructuración de relaciones cívicas que acercan diversos actores sociales, fomentan valores compartidos como responsabilidad, confianza, solidaridad, tolerancia y fortalecen el desarrollo de políticas públicas desde la intersectorialidad, para consolidar el desarrollo juvenil en un marco de equidad y participación.

\subsection{El reconocimiento de la juventud en las políticas como actor estratégico del desarrollo}

A partir del paradigma que reconoce la importancia de los jóvenes como actores estratégicos del desarrollo, se comienza a diseñar programas que puedan favorecer procesos de integración social para los jóvenes. Se sientan bases para el desarrollo de políticas de adolescencia y juventud incluyentes que se imbrican con la implementación del paradigma de ciudadanía juvenil. Esto hace relevante un claro enfrentamiento de la exclusión con el paradigma de la juventud como actor estratégico del desarrollo expresado en la articulación de las políticas públicas con un enfoque de transversalidad y equidad. 
Cuadro 5. Políticas avanzadas: paradigma de Actor Estratégico

\begin{tabular}{|c|c|c|c|}
\hline Paradigma & $\begin{array}{l}\text { Estrategias de } \\
\text { Políticas }\end{array}$ & Programas & Atención \\
\hline $\begin{array}{l}\text { Juventud: } \\
\text { Actor } \\
\text { estratégico } \\
\text { del desarrollo } \\
\text { Etapa de } \\
\text { formación } \\
\text { y aporte } \\
\text { productivo y } \\
\text { cultural }\end{array}$ & $\begin{array}{l}\text { Articuladas en } \\
\text { políticas públicas } \\
\text { Intersectoriales } \\
\text { Priorizan la } \\
\text { incorporación de las } \\
\text { juventudes como } \\
\text { capital humano, } \\
\text { desarrollo de capital } \\
\text { social y productor } \\
\text { cultural }\end{array}$ & $\begin{array}{l}\text { Transversalidad } \\
\text { institucional. } \\
\text { Empodera- } \\
\text { miento } \\
\text { Expresión } \\
\text { cultural } \\
\text { Aporte juvenil } \\
\text { al desarrollo } \\
\text { económico, } \\
\text { social y cultural }\end{array}$ & $\begin{array}{l}\text { Inclusión en el } \\
\text { desarrollo social } \\
\text { Fortalecimiento } \\
\text { del capital } \\
\text { humano, social, } \\
\text { cultural } \\
\text { Participación } \\
\text { efectiva } \\
\text { Enfrentamiento a } \\
\text { la exclusión } \\
\text { Reducción de } \\
\text { brechas sociales }\end{array}$ \\
\hline
\end{tabular}

Fuente: Dina Krauskopf. Nueva Sociedad \#200.2005. Versión modificada

En muchos países de América Latina, la pirámide demográfica todavía no se ha revertido, constituyéndose la población adolescente y juvenil en la ventana de oportunidad para un bono demográfico aún vigente, fundamento para procurar el incremento de las inversiones en el rescate del capital humano juvenil para resolver problemas del desarrollo en países que luego afrontarán una capa creciente de población mayor de edad que demandará más servicios y dependerá del aporte de los jóvenes de hoy a sus sociedades. Las distancias generacionales con los adultos se han modificado; ahora los jóvenes saben cosas que los adultos no saben ${ }^{3}$. Esto hace que la participación juvenil no solo sea un claro derecho, sino que también es una parte insustituible del conocimiento de las situaciones y alternativas (Krauskopf, 2003).

Las personas jóvenes, a menudo, proveen el ingreso principal de sus familias, trabajan tempranamente y en condiciones aza-

\footnotetext{
La falta de reconocimiento del nuevo balance muchas veces se expresa en los bloqueos generacionales (término aportado por el sociólogo uruguayo Julio Bango), una especie de discurso paralelo entre jóvenes y adultos que afecta incluso el sistema educativo y la vida familiar.
} 
rosas, superan la adversidad, aportan entusiasmo y creatividad. Migran masivamente hacia las ciudades en busca de mejores condiciones de vida y son marginalizados por un sistema cada vez más excluyente. Son los jóvenes los que se enfrentan con flexibilidad el desafío de las innovaciones tecnológicas y las transformaciones productivas, los que manifiestan sus sensibilidades en el arte y la cultura. Las políticas avanzadas de juventud se orientan a la formación del capital humano y social, la reducción de las brechas de desigualdad con una perspectiva de equidad y del aporte estratégico de las juventudes al desarrollo de su sociedad.

\section{La evolución de los paradigmas de salud y la adolescencia}

Congruente con los dos últimos paradigmas, propios de las políticas avanzadas en salud, la Organización Panamericana de la Salud propuso, en 1997, un modelo basado en el marco conceptual de salud y desarrollo para la promoción de la salud de adolescentes y jóvenes, que incorpora el enfoque de derechos con una estrategia intersectorial y participativa que genera sinergia entre los diversos componentes del desarrollo.

La evolución de los paradigmas de la salud ha dejado en evidencia que se trata de una construcción social. Las primeras definiciones siguieron una orientación médica y la salud se definió como una categoría residual, esto es, la ausencia de enfermedad. El impacto de este concepto en la atención de adolescentes es fundamental. Se infería que, al consultar escasamente por enfermedades, revelaban pertenecer a una 'edad sana'. Ello influyó para que estuvieran ausentes los servicios de adolescencia y predominaran los servicios del sistema de salud organizados para niños y adultos.

En 1978, la Declaración de Alma Ata evidenció la relación insoslayable entre salud y desarrollo. A partir de ello, se modificó la perspectiva médica de la salud y la Organización Mundial de la 
Salud estableció que se trata del estado de completo bienestar físico, mental y social y no solamente la ausencia de enfermedades. Más adelante, la Convención de los Derechos del Niño consideró la salud plena como un derecho humano fundamental. Por último, acorde con los fundamentos económicos que sustentan las inversiones en salud, esta es considerada un bien público.

\section{Cuadro 6. Evolución de los paradigmas de salud}

- Ausencia de enfermedades (salud: categoría residual)

- Estado de completo bienestar físico, mental y social

- Construcción social

- Desarrollo humano integral

- Salud plena: derecho humano fundamental

- Bien público. Pre-requisito para el desarrollo económico

Fuente: Dina Krauskopf. Elaboración propia

Es nuestro planteamiento que, en forma realista, la salud, más que un 'estado', como plantea la definición alcanzada en la Conferencia Internacional sobre Atención Primaria en Salud, celebrada en Alma Ata, es un balance entre diversos procesos biopsicosociales que permiten avanzar en dirección a la plenitud vital. Cuando predominan los procesos que comprometen dicho balance, se favorecen los daños a la vida. Por ello, OMS, OPS entienden la salud en la adolescencia como "un proceso continuo a través del cual el adolescente satisface sus necesidades, desarrolla competencias, habilidades y redes sociales" (1997:11). De esta forma, el desarrollo integral de los adolescentes y jóvenes aparece en el centro y es reconocido como una dimensión fundamental en su salud. El desarrollo humano se expresa en indicadores como el fomento del uso efectivo de capacidades y participación decisoria, niveles de educación, ingreso, ciudadanía y calidad de vida.

Ha sido difícil avanzar hacia una noción positiva y clara de la salud. Perry y Jessor (1985) observan que un concepto de esta naturaleza debería ir más allá no solo de la ausencia de enfermedad sino también de "la mera adecuación del funcionamiento 
personal y social" y señalan que la noción de salud está conectada con la idea de la totalidad de una persona (1985:170). Dan importancia a las ciencias comportamentales y sociales cuando destacan componentes como sensación de idoneidad, confianza, capacidad, compromiso social, contribución al mejoramiento de otros y realización de potencialidades individuales.

Para Perry y Jessor, es fundamental reconocer que la salud es un concepto diferente a la enfermedad; la confusión entre prevención y promoción proviene de una elusiva diferenciación de estos conceptos. Aclaran que la prevención de la enfermedad refleja el concepto de salud como ausencia de enfermedad; la promoción de la salud ha sido paulatinamente reconocida de un modo más claro e inclusivo. Puntualizan que la promoción incluye, además de estrategias para conservar la salud, el énfasis en acrecentarla. Por ello, la promoción de la salud está orientada, más que a la reducción o eliminación de comportamientos que comprometen la salud, a estimular prácticas que la enriquecen. Aquí, la consideración del contexto pasa a ser fundamental.

El valioso concepto de atención integral que plantea Elsa Moreno (1995): “...se refiere a la amplitud con que debe abordarse su atención. Este encuadre exige tener en cuenta no solo los factores biológicos sino también los estilos de vida, el ambiente físico y cultural y la oferta y organización de los servicios. El propósito fundamental de este enfoque es la preservación de la salud, estimulando la detección de riesgos y factores protectores y poniendo en marcha medidas que privilegien las actividades anticipatorias". Concordando con el marco conceptual y con las afirmaciones de Perry y Jessor, entendemos que es fundamental la promoción de la salud, ya que "emerge como una herramienta indispensable para el cambio social necesario para lograr el desarrollo y la salud de los adolescentes" (OPS-OMS-KELLOG, 1997:13). 


\section{Consideraciones finales}

El avance en los paradigmas sobre la fase juvenil y la salud han permitido que se inicien programas dirigidos a estas poblaciones que rompen su exclusión de los servicios y donde se reconoce que se trata de un período fundamental para la salud, el desarrollo humano y el desarrollo de las sociedades. Dicho abordaje requiere integralidad dentro del sector salud, pero también se torna indispensable la articulación intersectorial, la participación de los más diversos sectores de la sociedad junto al reconocimiento de la capacidad de actores estratégicos y ciudadanos de los propios adolescentes y jóvenes.

Para enfrentar el desafío de construir sociedades inclusivas, es necesario contar con legislación, políticas públicas, e institucionalidad orientadas al pleno reconocimiento de las capacidades y derechos de las juventudes y las medidas necesarias para su cumplimiento. Su realización es responsabilidad de las instituciones del Estado y del compromiso ciudadano de la sociedad y sus juventudes.

Una Política de Salud para la Adolescencia y Juventud es una Política Pública que define los lineamientos generales con visión compartida y estratégica para convertirse en el principal instrumento de referencia de las acciones dirigidas a la adolescencia y juventud, cuya salud y desarrollo pleno constituyen un componente básico para el desarrollo nacional. Debe traducirse en Planes de Acción con reorientación de los objetivos de los Planes operativos de los sectores que concreten de modo palpable las metas propuestas.

\section{Bibliografía consultada}

Beck, Ulrich (1998). La Sociedad de riesgo. Hacia una nueva modernidad. Buenos Aires: Paidós.

Blum, Robert (1996). Toward a New Millenium: A Conceptual Model for Adolescent Health. Washington, D.C.: Organización Panamericana de la Salud. 
Caja Costarricense de Seguro Social (2002). Bases programáticas. Programa de Atención integral a la Adolescencia. San José, Costa Rica.

ERIC; IDESO; IDIES; IUDOP (2004). Maras y pandillas en Centroamérica. Volumen II. San Salvador: UCA Editores.

Jessor, Richard (1991). "Risk Behaviour in Adolescence: A Psychosocial Framework for Understanding and Action". Journal of Adolescence Health. Nueva York.

Krauskopf, Dina (1996). "Violencia juvenil: Alerta social". En Revista Parlamentaria. La Crisis Social: Desintegración Familiar, Valores y Violencia Social. Vol. 4. No 3, 775-801, San José, Asamblea Legislativa de Costa Rica, 750-1215.

(2003). "Juventud, riesgo y violencia". En Dimensiones de la Violencia. El Salvador: Ed. PNUD.

(2003). Participación Social y Desarrollo en la Adolescencia (Tercera Edición). San José: UNFPA.

(2005). "Desafíos en la construcción e implementación de las políticas de juventud en América Latina". En El futuro ya no es como antes. Ser joven en América Latina. Noviembre-Diciembre, 141153, Buenos Aires, Argentina. Nueva Sociedad. 184 Páginas.

Moreno, Elsa (1995). "Los Servicios de Salud para los Jóvenes". Publicado en el Encuentro sobre Salud del Adolescente en Cartagena de Indias. Madrid: UNFPA OIJ -OPS.

OPS-OMS (1978). Declaración de Alma -Ata. Ginebra, Suiza.

OPS-OMS-Kellog (1998). Proyecto de desarrollo y salud integral de adolescentes y jóvenes en América Latina y el Caribe. Washington. OPS, OMS, Kellog.

Oyarzún, Astrid (2006). "De joven problema... A joven actor social estudiantil: estudiantes secundarios y su lucha por la igualdad educativa y social". En Gacetilla CIDPA. Valparaíso. CIDPA.

Perry, Cherril L. y Jessor, Richard (1985). "The Concept of Health Promotion and the Prevention of Adolescent Drug Abuse". En Health Education Quarterly. Ed. John Wiley and Sons, Inc.

Santa Cruz, María (2003). "Violencia y juventud en El Salvador". Presentado en la Conferencia Centroamericana de Juventud. Antigua, Guatemala: CIRMA, Rockefeller Foundation, Fundación Frederich Ebert.

Serna, Leslie (1998). “Globalización y participación juvenil”. En Jóvenes. Rev. de Estudios sobre Juventud. $4^{\mathrm{a}}$ Epoca. Año\#5. México. 